\title{
La segregación socio-espacial en dos ciudades turísticas costeras: Acapulco y Cancún
}

\section{The socio-spatial segregation in two costal tourist cities: Acapulco and Cancún}

\author{
Felipe de Jesús Moreno Galván* y Celia Hernández Diego"*
}

\section{Resumen}

Este trabajo tiene como objetivo analizar dos ciudades turísticas costeras: Acapulco y Cancún como ciudades que se configuran socio-espacialmente de forma segregada. Este estudio se desarrolla mediante la territorialización de datos estadísticos. Primero, se definen los distintos procesos históricos de urbanización de ambas zonas metropolitanas, bajo el reconocimiento de cómo se estructura, valoriza y define la dinámica turística en cada uno de ellos. En segundo término, se analiza la relación que se establece entre la configuración del espacio turístico, los índices de rezago social, los equipamientos y la densidad habitacional. Finalmente, se discute la idea que el turismo genera procesos complejos con efectos territoriales múltiples, que implican especializaciones funcionales, lo que a su vez refuerza un aumento de la segregación socio-espacial por aislamiento de las zonas hoteleras y las de vivienda y las tendencias al desarrollo de los nodos comerciales y corredores terciarios.

Palabras clave: Segregación socio-espacial, Estructura metropolitana, Tercerización, Servicios turísticos, Corredores terciarios.

\section{Abstract}

The objective of this work is to analyze two coastal tourist cities: Acapulco and Cancún as cities that are configured socio-spatially in a segregated manner. This study is developed through the territorialization of statistical data. First, the different historical urbanization processes of both metropolitan areas are defined, under the recognition of how the tourism dynamics are structured, valued and defined in each one of them. Secondly, the relationship established among the configuration of the tourist area, the social indexes, the equipment and the housing density is analyzed. Finally, the idea that tourism generates complex processes with multiple territorial effects, involving functional specializations, is discussed, which in turn reinforces an increase in socio-spatial segregation by isolation of hotel and housing areas and trends in the development of the commercial nodes and tertiary corridors.

Keywords: Socio-spatial segregation, Metropolitan structure, Tertiarization, Tourist services, Tertiary corridors.

Fecha de recibo: 05 de julio de 2018 • Fecha de aceptación: 13 de septiembre de 2018

* Profesor-Investigador del Departamento de Métodos y Sistemas de la Universidad Autónoma Metropolitana, Unidad Xochimilco, arsdhan@yahoo.com.

* Catedrática CONACyT-Instituto Tecnológico de Cancún, cehedi@hotmail.com.

Nota: Agradecemos la colaboración de Rafael Mora López por la elaboración de la cartografía usada en este documento. 


\section{Introducción}

El término "segregación" con frecuencia recuerda a los grupos sociales y zonas más pobres, sin embargo, en este documento se considera que es una condición estructural del funcionamiento de las ciudades contemporáneas por lo que implica a todos los sectores sociales. Para entender los procesos que definen la estructuración metropolitana es necesario, por tanto, diferenciar las causas de la segregación que en el caso de los grupos étnicos y los más pobres "se puede considerar consecuencia del rechazo de los sectores dominantes hacia esos grupos y del funcionamiento del mercado del suelo" (Schteingart, 2015, p. 49), en tanto que, los sectores dominantes y los participantes de los procesos económicos desarrollan una segregación activa. En este sentido, las ciudades turísticas costeras se organizan socio-espacialmente de forma segregada, ya que el funcionamiento de las actividades productivas en el modelo de los servicios turísticos dominante requiere la restricción selectiva de acceso a los atractivos turísticos como zonas de playa y escenarios naturales.

Estas tendencias tienen como rasgo estructurador la ubicación de servicios turísticos de alojamiento, alimentación y recreación, pero con características segregatorias desde su origen. En otras palabras, "el turismo en sí mismo implica, desde los orígenes históricos de esta actividad, la creación de espacios delimitados y apropiados para ejercitar la segregación social entre quienes pueden disfrutar de tiempos de ocio y los que cubren apenas sus necesidades básicas" (Castillo y Villar, 2011, p. 88). De esta forma, un factor clave presente en esta configuración socio-espacial reside en la comercialización del espacio turístico, sin embargo, existe otro conjunto de factores que refuerzan la existencia de un modelo segregatorio. Las afectaciones de los centros turísticos que llegan a una saturación, han sido estudiadas en otros casos y se ha observado que se generan patrones de expansión radiales, a lo largo de las rutas de transporte o siguiendo la forma de la costa. Esto genera afectaciones al paisaje natural y urbano, sobrecarga de infraestructura, segregación de residentes locales y congestionamientos (Mc Coy, 2015, p. 28).

Por lo tanto, no sólo el atractivo turístico ocupa espacios segregados sino que existen diversos niveles de prioridad como el requerido por otro conjunto de servicios turísticos complementarios, como los servicios personales, financieros, de transporte y el comercio (Pérez, 2010, p. 172). Además de estas actividades pueden encontrarse en la proximidad desarrollos inmobiliarios de vivienda de alto costo, que conforman en conjunto las denominadas "zonas hoteleras". Estas áreas funcionan como enclaves en el modelo contemporáneo y establecen límites físicos y virtuales para lograr zonas cuya exclusividad pueda ser también comercializada. Otro elemento que estructura la forma segregada de las ciudades turísticas lo constituyen las áreas que concentran los servicios, equipamientos y comercios; fundamentales para el soporte de los habitantes de las ciudades, áreas especializadas que funcionan a partir de las tendencias de reestructuración terciaria que afectan a la mayoría de las ciudades contemporáneas. En este sentido, las ciudades turísticas forman parte de una tendencia que afecta a otras ciudades con vocaciones productivas distintas, que se manifiesta en una preeminencia de las actividades terciarias, sobre todo, de comercio y servicios.

En este esquema, el costo de la vivienda y la distribución de los sectores sociales se encuentran vinculados a sus características internas como tamaño y calidad, acceso a los equipamientos y servicios y, de manera cada vez más relevante, a su exclusividad. La accesibilidad, por tanto, se convierte en un elemento clave para determinar el valor del suelo y, en consecuencia, la segregación de los distintos sectores sociales. Sin embargo, no se da sólo en una simple forma centro-periferia, ya que las estructuras urbanas contemporáneas no sólo se conforman a partir de la concentración y especialización de las actividades productivas, si 
no que presentan una estructura organizada por las vialidades de mayor capacidad y los sistemas de transporte, conformando áreas de concentración y especialización comercial y de servicios.

De igual modo, existen diversas condiciones objetivas que influyen en la segregación de las distintas clases sociales en el territorio urbano, sobre todo, mediante su influencia en el valor del suelo. Entre las que se pueden citar: La presencia de equipamientos y servicios, accesibilidad, condiciones de confort ambiental y nivel de vulnerabilidad. Además de estas variables se encuentran otras de índole cultural que asignan valores simbólicos a ciertos espacios en función del paisaje natural, imagen urbana, características de la comunidad, patrimonio histórico y, de manera reciente, la mercadotecnia que promueve la exclusividad como un nuevo valor que garantiza seguridad y el mantenimiento del valor inmobiliario.

En las regiones costeras mexicanas se despliega un conjunto de condiciones que puede representar riesgos para las poblaciones locales. De estas destacan los fenómenos hidrometeorológicos, la sismicidad y las enfermedades tropicales; acontecimientos que agudizan sus efectos en aquellos casos donde existen asentamientos en zonas de suelo inestable e inundable, generalmente áreas segregadas pobres. Por lo que es de la mayor importancia el entendimiento de los elementos estructuradores de la forma urbana para poder regular la existencia de áreas de mitigación e infraestructuras para garantizar una mayor resiliencia ante las diversas vulnerabilidades.

Para aproximarnos a un entendimiento de los mecanismos de la estructuración metropolitana, se tomaron dos casos de ciudades turísticas costeras como elemento de contraste; Acapulco es una ciudad con un largo desarrollo urbano de varios siglos y con una bahía adyacente a una sierra con pendiente abrupta, lo que crea un paisaje particular que sirve como uno de los atractivos turísticos del puerto. En tanto que Cancún es una ciudad diseñada y construida a partir de los setenta en un área continental plana, adyacente a una laguna y sobre una barra que también crea un escenario natural atractivo. Pese a sus diferencias, ambas son las ciudades turísticas más relevantes de México y es posible encontrar múltiples coincidencias en su forma de urbanización, su estructura urbana, sus patrones de segregación y sus vulnerabilidades. En este sentido, constituyen casos destacados de estudio, ya que su importante recepción de capitales turísticos no se ha traducido en una mayor capacidad de adaptación al medio, ni en políticas de desarrollo urbano y social.

\section{Metodología}

Esta investigación analiza comparativamente la segregación socio-espacial de Acapulco y Cancún, los usos de suelo y los sectores asentados en ellas, asumiendo que no puede existir una correlación automática entre los sectores segregados y variables aisladas, ya que la segregación socio-espacial está constituida por complejos procesos relacionados entre sí. Para plantear el desarrollo de las estructuras urbanas actuales se realiza en primer lugar una semblanza de su proceso de desarrollo urbano y de la industria turística, poniendo énfasis en su traza urbana y en la distribución histórica de los diferentes sectores. Posteriormente, para analizar las diferencias contemporáneas entre las zonas metropolitanas se utilizan índices de rezago social y se destacan sus vínculos con la distribución de los equipamientos. Otra variable que se analiza es la densidad habitacional para tratar de encontrar tendencias y relaciones entre el número de pobladores y los usos del suelo.

El índice de marginación urbana establecido por el Consejo Nacional de Población (CONAPO, 2010a) se basa en cuatro dimensiones: educación, salud, vivienda y bienes, en la versión de 2010 se construyó con indicadores en porcentajes sobre la población total; la población de 6 a 14 años que no asiste a la escuela, la población de 15 años o más sin educación básica completa, la población sin derechohabiencia a los servicios de salud e hijos fallecidos de mujeres de 15 a 49 años de edad. También se tomaron como indicadores los porcen- 
tajes de viviendas habitadas, sin drenaje conectado a la red pública o fosa séptica, sin excusado con conexión de agua, sin agua entubada dentro de la vivienda, con piso de tierra, con algún nivel de hacinamiento y sin refrigerador.

En tanto que el Consejo Nacional de Evaluación de la Política de Desarrollo Social (CONEVAL) utilizó en 2010 la metodología estadística de Análisis de Clases Latentes para crear un índice de rezago social utilizando los siguientes indicadores: población de 15 años y más analfabeta, población de 6 a 14 años que no asiste a la escuela, hogares con población de 15 a 29 años, con algún habitante con menos de 9 años de educación aprobados, población sin derechohabiencia a servicios de salud, promedio de ocupantes por cuarto. En cuanto a la vivienda se considera: los pisos de tierra, la falta de excusado, sanitario, agua entubada, drenaje y energía eléctrica; y en los indicadores de bienes se consideró la posesión de refrigerador y lavadora. Esta metodología permite diferenciar los distintos sectores sociales desde una perspectiva multidimensional, sin representar un estudio de la pobreza, ya que no incluye ingreso o alimentación, su mapeo permite una visión esquemática que contribuye a establecer la configuración espacial de los sectores segregados en esta investigación.

Adicionalmente para los mapas desarrollados, en este documento el equipamiento se obtuvo a partir de la información geoestadística de junio de 2016 denominada "Marco geoestadístico nacional", compilada en la Cartografía geoestadística urbana y rural amanzanada del Instituto Nacional de Estadística y Geografía (INEGI). En tanto que los mapas de densidad fueron de elaboración propia basados en el Censo de población y vivienda 2010 a escala de Áreas geoestadísticas básicas (Ageb) Urbanas y con una relación entre la población y el tamaño de cada Ageb.

Con los índices de segregación presentados geográficamente, combinados con el análisis del equipamiento y de las densidades, es posible establecer a nivel general los esquemas contemporáneos de segregación de ambas ciudades. Esta información se analiza comparativamente para encontrar tendencias comunes y diferencias, tanto en el desarrollo urbano como en la forma en que la industria turística se vincula con el medio y sus efectos en la calidad de vida y la vulnerabilidad de las zonas metropolitanas de Acapulco y Cancún.

\section{Proceso de urbanización de la Zona Metropolitana de Acapulco}

En Acapulco existen tres zonas turísticas que se han ido desarrollando con sus propias dinámicas históricas, socioeconómicas y territoriales; cada una con potenciales de atracción turística que inciden en la reestructuración local y en la generación de múltiples redes a diferentes escalas. En tanto que, las zonas habitacionales se han ubicado en una primera etapa, integradas a las zonas turísticas y, posteriormente, en una periferia extendida. La importancia del estudio de estas zonas radica en que permite reconocer distintas configuraciones territoriales y regionales al reflejar momentos históricos particulares en sus conformaciones urbanas y arquitectónicas. En este sentido, se pueden distinguir tres zonas con momentos históricos definidos (Zona Tradicional, Zona Dorada y Zona Diamante) (Ver Mapa 1).

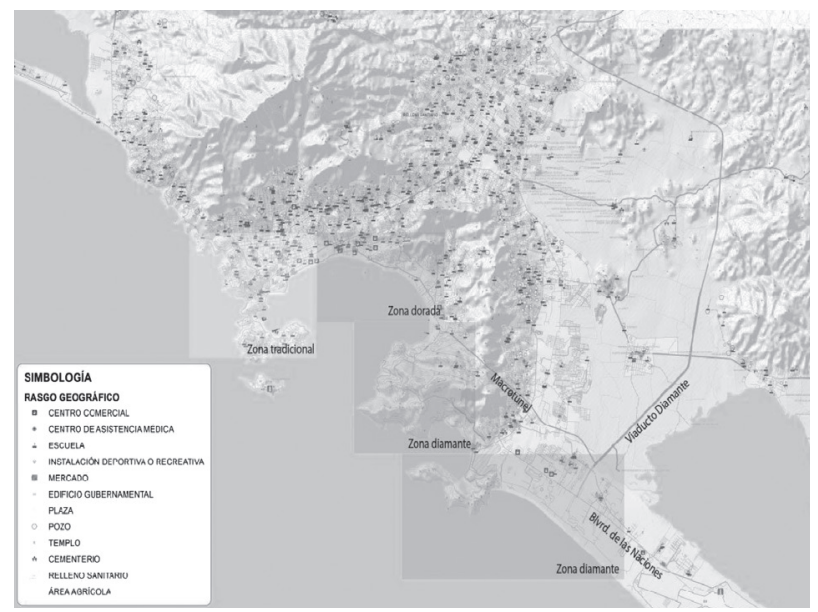

Mapa 1. Esquema general de la Zona Metropolitana de Acapulco con equipamientos principales. Fuente: elaboración propia con base en INEGI (2016) 
En Acapulco existen tres zonas turísticas que se han ido desarrollando con sus propias dinámicas históricas, socioeconómicas y territoriales; cada una con potenciales de atracción turística que inciden en la reestructuración local y en la generación de múltiples redes a diferentes escalas.

LaZona Tradicionalse desarrolló en las proximidades del antiguo centro de Acapulco, el puerto y el fuerte de San Diego, importantes durante la época virreinal debido a la ruta entre Asia, Europa y América, misma que utilizaba el puerto de Veracruz en el Golfo de México y Acapulco en el Pacífico como paso en la ruta entre Manila en las Filipinas y Sevilla en España. Después de la Independencia, esta ruta quedó cancelada disminuyendo notablemente la importancia de Acapulco.

Es hasta el año 1900 cuando Acapulco empieza a resurgir como puerto de reabastecimiento de la ruta San Francisco-Panamá. Este fenómeno se reforzó cuando en 1927 fue inaugurada la carretera Acapulco-Taxco-Cuernavaca-Distrito Federal, lo que ocasionó la llegada de turistas y la consecuente demanda de servicios e infraestructura. Es por tanto, en los treinta cuando se inician las construcciones sobre la Península de las Playas, constituyendo la zona de Acapulco Tradicional desde la Isla Roqueta hasta lo que ahora es el Parque Papagayo, es notorio que de 1930 a 1940 la población aumentó un $35 \%$ al pasar de 6,529 a 9,993 habitantes (INEGI, 1960). Sin embargo, no fue hasta el periodo presidencial de Miguel Alemán (1946-1952) cuando el gobierno fomentó el desarrollo turístico de toda la Bahía de Acapulco. Es entonces cuando se trazaron la calzada Costera y la gran vía tropical, se trasladó el aeropuerto hacia las afueras de la bahía y se construyó la autopista que conectaba el Distrito Federal con Acapulco (Gormsen, 1977, p. 37). Además se expropiaron terrenos a lo largo de la bahía, se tendió la red telefónica y apareció una infraestructura turística diversificada y moderna, con numerosos restaurantes, comercios y servicios que satisfarían las necesidades de la población demandante.

Los primeros datos estadísticos disponibles sobre turistas y número de cuartos de hotel se registraron hasta 1954, año en que hubo 92,694 turistas (Gerstenhauer, 1956, p. 278, citado en Gormsen, 1977, p. 38). Particularmente, entre 1950 y 1960 Acapulco se dio a conocer internacionalmente, con el surgimiento de una moderna infraestructura cerca de las playas de Caleta y Caletilla y el aumento de los medios transporte para satisfacer la demanda de servicios, principalmente por vía aérea y terrestre (Galván, 2000). Así empezaron a aparecer consorcios de servicios turísticos para atender a los cada vez más grandes flujos de turistas. Esto también propició flujos migratorios tanto de las regiones internas del estado como de otras entidades federativas. Mientras Acapulco fortalecía sus redes externas, las internas comenzaban a mostrar fracturas de tipo socioterritorial y ecológico.

Aunque oficialmente no existe una definición categórica del tiempo, espacialidad y límites de la segunda etapa, su duración se reconstruye a partir de las evidencias arquitectónicas, infraestructura, cambios en los planes de desarrollo y muy particularmente la percepción de actores involucrados en su nacimiento y consolidación. Esta segunda etapa tiene como escenario lo que hoy se conoce como la Zona Dorada de Acapulco. Geográficamente comprende desde los límites del Parque Papagayo hasta la Base Naval Militar. En la actualidad, se caracteriza por la modernidad de sus instalaciones, accesibilidad y cercanía a discotecas, restaurantes, centros comerciales, diversiones de playa y nocturnas, etcétera. Después de los cambios en la década de los cuarenta y cincuenta, en términos de conectividad y accesibilidad al puerto, la morfología de Acapulco empezó a transformarse. El turismo se entrelazó con el desarrollo urbano, 
la comunidad local y la población migrante. De tal forma que la construcción de hoteles, condominios y mejora en las vías de comunicación local se dio de manera creciente a lo largo de la franja costera.

Los primeros datos estadísticos oficiales se registran a partir de 1960, donde se tuvieron 540,100 visitas, lo que representó un aumento de casi 6 veces más del número de turistas con relación a $1954(92,694$ visitantes) (INFRATUR, 1973; CIETAL, 1991, p. 12). Para 1963 se pavimentó completamente la carretera, lo que condujo a una mayor accesibilidad al puerto, motivando así, la llegada masiva de turistas desde diferentes puntos geográficos (Gerstenhauer, 1956, p. 275 citado en Gormsen, 1977, p. 36). Al reducirse el tiempo de traslado los turistas podían desplazarse en sus propios autos, aumentando el turismo proveniente tanto de la ciudad de México y lugares aledaños como de los que venían de Estados Unidos, vía carretera.

En efecto, el número de visitantes nacionales y extranjeros fue importante para la economía del puerto, esto se ve reflejado en los datos de 1964 cuando Acapulco representó el 40\% de las rentas fiscales del estado del orden de los 40 millones de pesos (Ochoa, 1964, p. 105). Para mediados de los años sesenta las estadísticas nuevamente son favorables, al mostrar un aumento considerable del número de visitantes debido probablemente a la puesta en servicio del nuevo aeropuerto de Acapulco que inició operaciones con equipo jet en 1967 y cuyos datos hacen referencia a un arribo de 81,000 personas.

Además de las mejoras en las vías de acceso terrestres y aéreas, entre 1969 y 1971 se pusieron en servicio varios hoteles de gran capacidad, logrando un crecimiento en el número de cuartos que va de 4,694 en 1960 a 12,379 en 1971, (Schornick, 1977, p. 40). En conjunto, tales cambios tuvieron un reflejo en la economía; tan sólo para 1970 la aportación del turismo en el PIB nacional fue del 26\%, superando al producido por la industria manufacturera (INEGI, 1987) y, asimismo, en el ámbito local la aportación del turismo al PIB del estado de Guerrero fue del $30.4 \%$.
De tal forma que en 1970 Acapulco alcanzó su auge en términos competitivos, al grado que los turistas en menos de cinco años ocupaban casi completamente la bahía, lo que provocó serios problemas ambientales, viéndose afectada la imagen "paradisíaca" por la imagen "moderna" de Acapulco (Galván, 2000). La demanda fue tan grande que provocó un desequilibrio en la imagen arquitectónica, ya que no existía una reglamentación clara de dónde construir, del cuidado ecológico de las construcciones y su entorno. De tal forma que la anarquía y la poca planeación territorial fue deteriorando las características geográficas originales de Acapulco.

Aunado a lo anterior, en 1972 se presentó un claro estancamiento en materia de construcción (Schornick, 1977, p. 40); fenómeno que afectó en forma evidente los ingresos fiscales originados por los diversos rubros turísticos en los que directa o indirectamente incidía este sector económico. Tan sólo para 1967 representó el 11.5\% (4,437 millones de pesos) y en 1968 el 10\% (3,582 millones de pesos) del total de entradas fiscales al país. No obstante, el panorama no fue del todo desfavorable: a pesar de las adversidades socio-económicas, para 1972 los turistas vía aérea aumentaron a 247,000 de una cifra total de 1.5 millones de llegadas (INFRATUR, 1973, p. 23, citado en Gormsen, 1977, p. 40) y para 1979 el turismo seguía superando al petróleo en la captación de divisas, la relación era de 101,433 y 87,157 millones de pesos respectivamente, donde Acapulco participaba con el $42 \%$.

Por lo tanto, se puede señalar que a finales de los setenta en Acapulco se tenían dos condiciones encontradas. Por un lado, un saldo favorable en la economía, pero por otro, un desgaste de los atractivos turísticos y su inicial estancamiento. A estos fenómenos se sumó el nacimiento de los centros integralmente planeados como competidores inmediatos en materia turística, lo que provocó que las ventajas comparativas y competitivas con las que se venía desarrollando Acapulco aminoraran de manera notable. Desde este momento se tuvieron que buscar nuevos mecanismos para 
mejorar las condiciones de competencia y competitividad a otra escala. De esta manera, para 1980 las edificaciones se empezaron a planear con una imagen moderna y con diseños más atractivos, pero a pesar de los esfuerzos de inversionistas y de las autoridades gubernamentales, los atractivos turísticos comenzaron a dar muestras de desgaste. Así, entre finales de la década de los setenta y ochenta, en casi todas las variables turísticas se presentaron tasas de decrecimiento. Una de las primeras muestras se reflejó en el número de vuelos, desplazamiento de vehículos, turistas extranjeros, número de cuartos, etcétera.

En la década de los noventa se tuvieron que tomar medidas para cambiar la imagen del puerto. Se inició una remodelación de la Zona Tradicional, en la Dorada hubo un replanteamiento de avenidas, reforestación de áreas verdes en las calles más cercanas a la avenida costera, se reubicaron comercios y vendedores ambulantes, se hizo mayor limpieza de las calles y playas, se mejoró la imagen urbana-turística, se invitó a la población a tomar conciencia de la importancia del turismo en la economía local, hubo incentivos a inversionistas, etcétera. Asimismo, surgieron las primeras intenciones de hacer un nuevo proyecto turístico, pero ahora con mejores niveles de planeación y una estructura pensada a largo plazo.

En los noventa fue el periodo cuando se transforma con mayor fuerza el proyecto "Acapulco Diamante", lo que es hoy la tercera área turística de Acapulco llamada Zona Diamante. Ésta se ubica desde la Carretera Escénica hasta el área de Barra Vieja, zona antes conocida como "Punta Diamante"; misma que ha crecido más allá de los diez millones de metros cuadrados originales y que incluye: áreas de descanso, vida nocturna, playas con todos los servicios, una de las infraestructuras hoteleras más grandes de Latinoamérica, marina, campos de golf, además de la cercanía a la Autopista del Sol que conecta con la ciudad de México.

La Zona Dorada fue diseñada como un enclave turístico, la zona más exclusiva, con la mejor infraestructura técnica, informacional y de acceso.
Se creó con la intención de hacer una reconvergencia en el territorio y dar una nueva alternativa al turista. Si bien, por un lado abrió una nueva oferta al consumo, por el otro también la cerró, debido a que no todos los turistas tienen la posibilidad de pagar el precio alternativo. De hecho, ni siquiera pueden hacer un consumo inmaterial del paisaje o de las playas, pues el acceso también se encuentra restringido y amurallado.

Las redes en este nuevo espacio son de gran dinamismo, tecnológicamente superiores, con mayor proyección internacional, pero, al mismo tiempo, selectivas y excluyentes; una muestra significativa de la realidad globalizante. Lo importante aquí sería rescatar en lo posible, la metamorfosis de las zonas turísticas, el proceso que las explica y reconocer las implicaciones de tener nuevas zonas turísticas, no sólo en términos geográficos, sino desde el punto de vista socio-territorial, para entender que el ocio en buena medida también fortalece las diferencias y las legitimiza en sus prácticas.

\section{Proceso de urbanización de la Zona Metropolitana de Cancún}

Como demostró Acapulco, México poseía importantes potenciales para el desarrollo turístico, pese a esto hasta la década de los sesenta no se habían desarrollado otros destinos, así que en 1968 el Gobierno Federal cedió la política de desarrollo turístico al Banco de México el cual había establecido desde 1934 la Banca Nacional Financiera de México (NAFIN) una institución filial para fomentar y financiar las empresas industriales. En 1974 fue creado el Fondo Nacional de Fomento al Turismo (FONATUR), "el cual colabora con los objetivos de NAFIN desarrollando, planificando y financiando la actividad turística nacional" (Aldape, 2010, p. 12). Los primeros proyectos de FONATUR partieron de una política de desarrollo de polos turísticos que fungieran como impulsores del desarrollo en el oriente de la Península de Yucatán, al sur de la Península de Baja California y 
en la costa sur del país, regiones con bajos niveles de desarrollo económico y social (Castillo y Villar, 2011, p. 92) de esta manera los Centros Integralmente Planeados (CIP) fueron inicialmente: Cancún, Los Cabos, Ixtapa, Huatulco y Loreto; proyectos en los que FONATUR funcionó como un gestor de acciones que incluyeron: "solicitud y garantía de fondos a la banca de desarrollo, adquisición de tierra, diseño y construcción de infraestructura, promoción comercial de lotes urbanizados e integración de recursos para finiquitar deuda pública" (Aldape, 2010, p. 15).

La planificación de los CIP se plasmó en la forma de planes "maestros", entre los cuales Cancún sentó precedente ya que se realizó a partir de un análisis de sitio y de su región, también se establecieron matrices para establecer las afinidades entre usos del suelo tanto en la zona turística como en la zona urbana y de los usos contra factores ambientales (FONATUR, 1982), Cancún comenzó su construcción en 1970 y empezó su operación en 1974, el polígono original del enclave comprende una parte de la placa continental, una cuenca lagunar y una barra paralela a la costa, con corrientes subterráneas debido a la permeabilidad de las formaciones calcáreas, al igual que el resto de la península de Yucatán.

El Plan director de Cancún concibió a la ciudad en tres áreas diferenciadas: la turística sobre la duna, la residencial y de servicios, sobre la placa continental y la del aeropuerto en la misma placa continental al sur de la zona habitacional (Martí, 1985, p. 27). En particular, la zona turística sobre la duna se concibió como un "corredor turístico" contenido en sí mismo para albergar las instalaciones hoteleras, condominios, centros comerciales, campos de golf, las marinas y el centro de convenciones (Aldape, 2010, p. 43). La barra coralina se caracteriza por dos grandes puntos de articulación: la Punta Cancún al Norte, sitio de ubicación de comercios y centros de espectáculos y la Punta Nizuc al sur. El desarrollo de la zona turística se planificó en tres etapas, la primera etapa aumentaba progresivamente la densidad e
La planificación de los CIP se plasmó en la forma de planes "maestros", entre los cuales Cancún sentó precedente ya que se realizó a partir de un análisis de sitio y de su región, también se establecieron matrices para establecer las afinidades entre usos del suelo tanto en la zona turística como en la zona urbana y de los usos contra factores ambientales.

importancia de los hoteles desde la zona urbana hasta el espacio entre la Punta Cancún y la Playa Marlín. La segunda etapa se planeó desde Playa Marlín hasta la Caleta Nizuc con una densidad promedio de 120 cuartos/ha. En este esquema se destaca que el frente hotelero se representa como una barrera que ocupa el territorio de privilegios, privatizando el espacio costero, como destaca Aldape (2010, p. 64). En primera instancia se consideró que los hoteles proveerían de servicios de menor tamaño a sus huéspedes, con una oferta de restaurantes, comercios y equipamientos como centros de convenciones independientes de los hoteles, para promover la salida de los turistas a los centros comerciales y culturales.

El proyecto establece la separación de la zona turística de la zona habitacional y de servicios ubicada en la parte continental, misma que se estableció en el espacio ocupado entre dos ejes principales, la carretera a Mérida y la carretera a Chetumal, esta zona se diferenció en un núcleo portuario, uno urbano y una de industria ligera al oeste cercano a la salida a Mérida. En la zona habitacional se establecen tres densidades, la baja hasta 120 hab./ha; la media de 160 hab./ha. y una densidad alta para los habitantes de bajos recursos desde 200 a 300 hab./ha. En su primera etapa la zona habitacional estableció una traza de supermanzanas, mediante 
la agrupación de varias manzanas con áreas verdes y circulaciones locales cerradas a su interior, en tanto que estas supermanzanas se encuentran circundadas por avenidas de flujo vehicular intenso. Este modelo orientado al automóvil generó una ciudad hostil para el peatón, dado que las amplias curvas, incorporaciones y camellones facilitan la circulación vehicular en la zona de las supermanzanas pero impiden la circulación a pie y con bicicleta.

Este espacio original se desbordó desde su nacimiento, como resultado, se presentaron flujos migratorios de trabajadores de la construcción atraídos por las masivas inversiones inmobiliarias (Castillo y Villar, 2011, p. 93). A esta oleada se sumaron los trabajadores de la hotelería, con un nivel de ingreso que no les permitió acceder a las viviendas de la zona planificada esto de acuerdo a Castillo y Villar (2011, p. 93) favoreció el crecimiento de nuevos asentamientos irregulares en Puerto Juárez, el ejido Bonfil y la zona norte de Cancún.

Más al sur el aeropuerto se ubicó cercano a la carretera a Chetumal y al acceso sur de la duna, también denominada la isla de Cancún, lo que dota a la zona turística de un acceso inmediato desde la terminal aérea y permite, asimismo, una salida directa al desarrollo de infraestructura hotelera lineal sobre la carretera a Chetumal que está creando una conurbación entre los asentamientos de Cancún, Puerto Morelos y Playa del Carmen.

\section{La estructura urbana} contemporánea de Cancún se encuentra desarticulada dadas las diferencias entre las zonas turística y residencial y las denominadas "regiones", áreas de asentamiento populares en donde se ubica hasta un $80 \%$ de la población.
La construcción de Cancún incluyó obras de infraestructura como la ampliación de Puerto Juárez, un sistema de electricidad, alumbrado, teléfono, agua potable y drenaje para una población estimada en 40,000 habitantes (FONATUR, 1982:19). Sin embargo, esto no atrajo las inversiones hoteleras de manera inmediata por lo que FONATUR debió promover los proyectos iniciales con paquetes de proyecto, terreno y crédito (Aldape, 2010, p. 58).

Para 1987 la superficie urbana ocupaba un total de 1,285 hectáreas en las que se alojaba una población de 168,000 habitantes (Castillo y Villar, 2011, p. 93) por lo que las áreas previstas en el plan anterior habían sido rebasadas, lo que generó la producción de asentamientos irregulares en terrenos ejidales al norte de la ciudad, a esta área se le regularizó y estableció una traza reticular y ejes viales lineales a partir del Plan de 1993.

En el Programa de Desarrollo Urbano del Centro de Población de la Ciudad de Cancún de 2005 (Ayuntamiento de Benito Juárez, 2005) se establecen las problemáticas de la falta de áreas verdes con un promedio de $2,9 \mathrm{~m} 2$ por habitante y el cambio de uso de suelo en diversas supermanzanas céntricas. En este programa se regularizaron las zonas habitacionales surgidas al norte y poniente. En general, se ha observado una elevación de la densidad constructiva en la zona turística a partir del Plan de desarrollo de 1993 que afecta en consecuencia el crecimiento de la zona habitacional (Ayuntamiento de Benito Juárez, 1993). En este sentido, el constante incremento de la oferta hotelera incide directamente en el crecimiento de la zona urbana, tanto por aumento de la demanda de trabajadores como por la conversión de Cancún en un centro logístico para la Riviera Maya (Calderón y Orozco, 2009, pp. 30-31).

De acuerdo a Castillo y Villar (2011, pp. 93-94) el patrón de desarrollo de Cancún se organiza a partir de fraccionamientos de vivienda unifamiliar y vivienda dispersa en la zona noroeste de la ciudad y el ejido Alfredo V. Bonfil, un proceso simultáneo a la construcción de fraccionamientos privados para la población de altos y medianos 
ingresos en la primera periferia al suroeste de la zona centro y en partes de la zona turística. En una década la ciudad se expandió de 2,420 hectáreas en 1990 hasta 4,057 hectáreas en 2000. Esta fase de expansión estuvo acompañada de desarrollos inmobiliarios, equipamientos y servicios en las vialidades paralelas Tulum y Bonampak que conformaron un corredor urbano con departamentos residenciales y oficinas para corporativos de lujo. El resultado de este proceso, que alcanza ya cerca de cinco décadas,

...fue definiendo sus rasgos característicos desde los primeros años del desarrollo de Cancún enmarcados por la existencia de una aglomeración urbana integrada por tres "ciudades" colindantes, diferenciadas urbanística y socioeconómicamente, y claramente segregadas social y funcionalmente: una para los turistas, otra para sectores medios y altos ingresos y la tercera para los trabajadores de menores ingresos (Castillo y Villar, 2011, p. 94).

El ingreso a la economía neoliberal en la década de los noventa coincidió con la ampliación de las áreas de turismo: la denominada Riviera Maya, misma que se desarrolló con la apertura a la inversión extranjera (Segrado et al., 2010, p. 29). Sin embargo, pese a la ocupación de la franja litoral el resto del estado de Quintana Roo presenta aún niveles muy bajos de urbanización.

La estructura urbana contemporánea de Cancún se encuentra desarticulada dadas las diferencias entre las zonas turística y residencial y las denominadas "regiones", áreas de asentamiento populares en donde se ubica hasta un $80 \%$ de la población (Espinosa, 2013, p. 162). De manera particular, en Cancún se han establecido diversos grupos socioeconómicos provenientes de migraciones intra estatales, nacionales e internacionales, lo que da una dimensión adicional al problema de la segregación al establecerse relaciones entre las etnias de procedencia, sus ocupaciones, sus características demográficas y sus ubicaciones territoriales (Ver Mapa 2). En términos generales, el proceso de urbanización

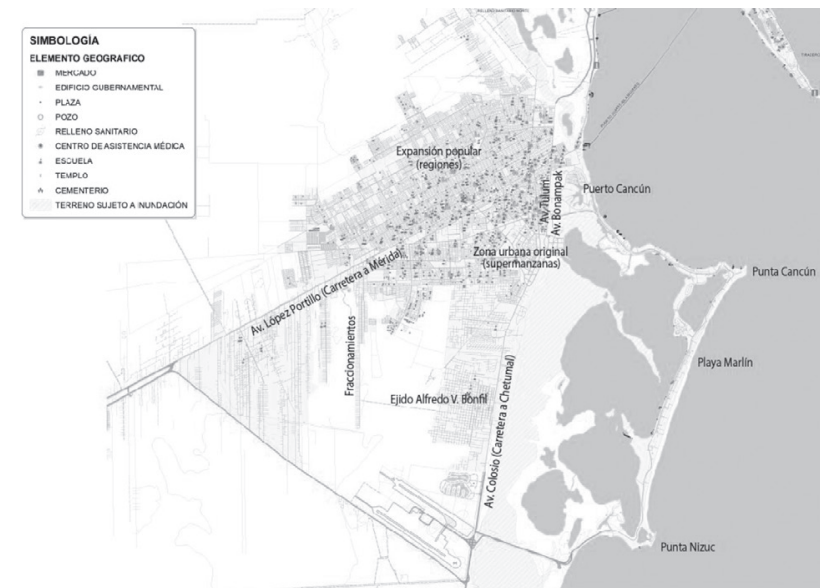

Mapa 2. Esquema general de la Zona Metropolitana de Cancún con equipamientos principales. Fuente: elaboración propia con base en INEGI (2016)

de Cancún ha consumido espacios naturales y paisajes conformando un sistema lineal urbano costero que se ubica en la franja litoral (Calderón y Orozco, 2009, p. 29); sistema metropolitano que no ha logrado integrarse económicamente con su región, reflejando la segregación a escalas regionales y confirmando el fenómeno ya observado en otros polos turísticos, separados de su área de influencia (Espinosa, 2013, p. 160).

\section{Configuración del espacio turístico y segregación socio- espacial}

Es notorio que las principales zonas periféricas populares de Acapulco y Cancún han tenido un gran crecimiento ya que la industria turística requiere una gran cantidad de mano de obra, en general poco especializada para funciones de mantenimiento y servicios básicos, y dado que los enclaves turísticos en México se encuentran en áreas originalmente poco pobladas, se han generado importantes flujos migratorios con la consecuente expansión de las áreas urbanas por adición de asentamientos populares.

En el proceso histórico de urbanización de estas ciudades, resulta lógico que las áreas más favo- 
recidas por su accesibilidad, seguridad y paisaje hayan tenido preferencia para los asentamientos y en el transcurso del tiempo concentren los principales equipamientos y sectores de ingresos altos. Sin embargo, también se observa el surgimiento de nuevos desarrollos para sectores de ingresos altos sobre zonas de difícil urbanización y mantenimiento como las áreas pantanosas de los manglares y el modelo de expansión que afectó a otras ciudades mexicanas a partir de grandes desarrollos de vivienda producida por empresas privadas. Este modelo reduce estadísticamente los índices de segregación debido a que existe una mayor combinación de clases sociales en los espacios periféricos, sin embargo, a largo plazo afecta a la vivienda popular como menciona Sabatini:

Resulta paradójico que esta virtual expulsión de la vivienda social sea el resultado de los procesos de dispersión de proyectos residenciales de clases medias y altas en la antigua periferia popular, esto es, consecuencia de la reducción de la segregación residencial. La llegada a la periferia popular de estos 'mejores pagadores' por el suelo, en la jerga económica, ha revolucionado las expectativas de los propietarios locales de terrenos. El precio al que están dispuestos a vender sus tierras se ajusta, por expectativas (o lo que no es sino lo mismo, por especulación), a la capacidad de pago de los nuevos moradores del área. Así, el suelo a precio compatible con la construcción de vivienda social tiende a desaparecer (Sabatini, 2016, p. 444).

En este sentido las ciudades estudiadas son afectadas al igual que otras por los cambios económicos macroestructurales, que afectan el mercado del suelo y la inversión inmobiliaria, lo que se refleja en el desarrollo de configuraciones territoriales como corredores terciarios a lo largo de las vialidades principales que agrupan comercio y servicios. Adicionalmente, en estas ciudades se crean zonas especializadas que no existen en otras, tales como las áreas destinadas a las actividades turísticas que operan de manera distinta y separada al resto de la ciudad.

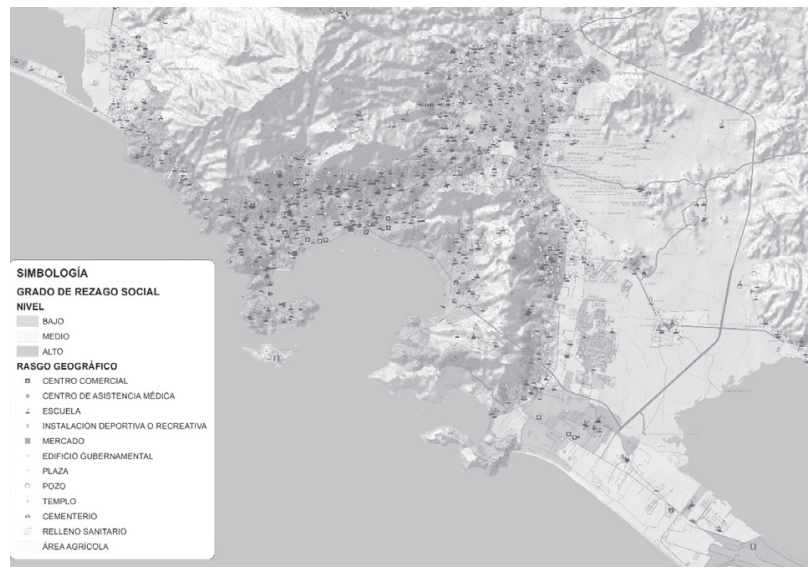

Mapa 3. Zona Metropolitana de Acapulco, grado de rezago social 2010. Fuente: elaboración propia con base en CONEVAL (2010)

En Acapulco debido a su proceso de urbanización desarrollado durante largo tiempo, las áreas más antiguas y consolidadas presentan un nivel de equipamiento más elevado con lo que tienen un bajo grado de rezago social, al mismo tiempo que las áreas periféricas recién urbanizadas y en zonas de difícil acceso como las laderas de la serranía presentan grados de rezago social más altas.

Es notorio que el grado de rezago social no tiene una correlación con la cercanía y densidad de los equipamientos en las zonas de ingresos altos; ya que el grado de rezago social no se construye a partir de la cercanía de los equipamientos sino de la capacidad de acceder a sus servicios. Esto es especialmente visible en la Zona Dorada y en áreas de la Zona Diamante, ya que las clases medias y altas acceden a los satisfactores mediante una mayor capacidad de movilidad, por lo que sus zonas residenciales resultan más homogéneas en términos de usos del suelo, dedicado especialmente como áreas residenciales, excluyendo otros tipos de actividades. En tanto que en las zonas de rezago social alto, si tiene un impacto la carencia de equipamientos y servicios, dado que esa población presenta una capacidad de movilidad menor y requiere la cercanía de los equipamientos para poder acceder a ellos. Para Sabatini (2015, p. 27) hay un cambio en el patrón de segregación que 
genera un "efecto gueto" en muchos barrios populares, esto es especialmente notorio en las zonas topográficamente más altas y de difícil movilidad (Ver Mapa 3).

En los esquemas de desarrollo hotelero contemporáneo las áreas turísticas resultan excluyentes de otros tipos de uso del suelo, por lo que no se observan equipamientos en las áreas litorales de nuevo desarrollo, especialmente en la zona diamante. En contraste, la zona tradicional y dorada integran los hoteles en zonas de uso de suelo heterogéneas, en parte esto se explica porque el modelo hotelero que estableció la zona tradicional y dorada no operaba aún bajo el principio de ser autosuficiente. Es decir, los hoteles más antiguos no poseían todos los servicios en su interior, dedicándose específicamente al alojamiento, dejando los servicios restauranteros y de recreación a otros proveedores; lo que implicaba una mayor relación de los turistas con otros prestadores de servicios locales y, por tanto, una mayor derrama económica hacia los agentes locales.

Tales fenómenos generaron áreas diversas, en las cuales, en distancias cortas a las zonas hoteleras tradicionales se presenta una variedad de servicios complementarios, caracterizados por una oferta variada y de precios razonables, además de los servicios propios de las áreas urbanas, situación que ha logrado aumentar la mortandad competitiva de estos espacios ante la falta de una distribución equitativa del mercado turístico. En total contraste con las fórmulas innovadoras de comercialización de los nuevos desarrollos turísticos que funcionan separados del resto de la estructura urbana y que, mantienen cautivos al turista y monopolizan la derrama económica del sector con toda clase de ofertas complementarias, tales como: servicios integrados de entretenimiento, centros comerciales, venta de artesanías, restaurantes, limpieza y planchado de ropa, medios de transporte hotelaeropuerto, etcétera.

En el caso de Cancún las áreas están claramente zonificadas desde su concepción, por lo que el área urbana concentra los equipamientos reque-

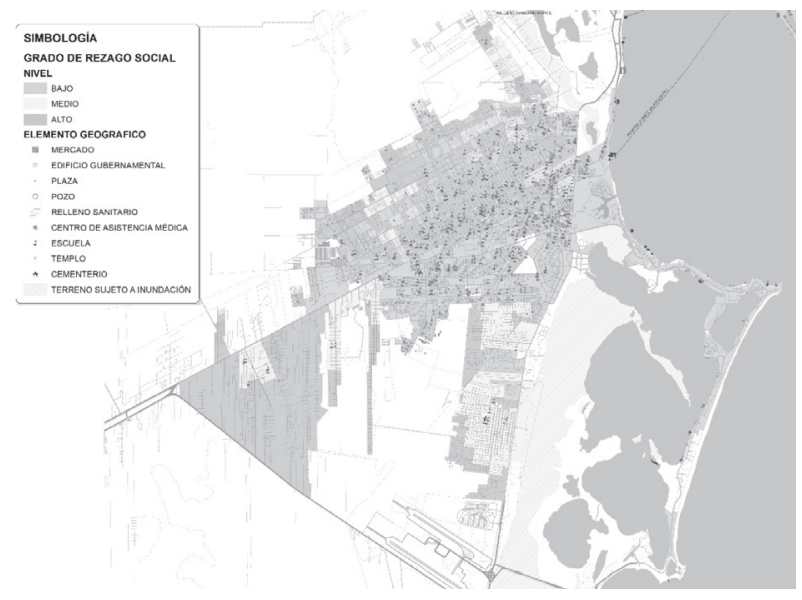

Mapa 4. Zona Metropolitana de Cancún, grado de rezago social 2010. Fuente: elaboración propia con base en CONEVAL (2010)

ridos para la reproducción de la población local, se observa un esquema centro-periferia donde las áreas más alejadas del área habitacional original resultan también con los grados de rezago social más altos. También es notorio el proceso de urbanización reciente de las regiones de producción popular y de desarrollos de interés social al norte de la ciudad, ya que se observa un marcado decrecimiento del número de equipamientos. De igual manera se observa esta carencia en el ejido Alfredo V. Bonfil, mismo que fue creado desde la fundación de la ciudad y que actualmente todavía carece de muchos equipamientos y servicios aunque se encuentra en el camino de la expansión inmobiliaria de la ciudad (Ver Mapa 4).

Pese a estar en una zona ambientalmente privilegiada, paradójicamente las áreas populares de Cancún cuentan con pocas áreas verdes y espacios públicos. Condición que se combina con una estructura vial desarrollada en mayor medida para los vehículos particulares y la existencia de grandes desarrollos comerciales y fraccionamientos cerrados, lo que crea una estructura urbana poco permeable que afecta a la movilidad y a la habitabilidad de la ciudad. Al igual que en Acapulco Diamante, donde los nuevos desarrollos hoteleros son excluyentes de otros tipos de uso de suelo y se encuentran orientadas al turismo masivo de sol y 
playa, con una oferta de servicios restauranteros y de recreación dentro de los mismos macrocomplejos hoteleros, lo que fragmenta aún más el funcionamiento de los sistemas urbanos.

Un aspecto a destacar es la distribución de la población que este esquema genera, la Zona Metropolitana de Acapulco tenía una población en 2010 de 863,431 habitantes y una tasa de crecimiento media anual de $0.8 \%$ en $3,538.5 \mathrm{~km}^{2}$, lo que correspondió a una densidad media urbana de 98 hab./ha. En tanto que la Zona Metropolitana de Cancún tenía una población de 677,379 habitantes, una tasa de crecimiento media anual del 4.5\% en una superficie de $3,053.6 \mathrm{~km}^{2}$, y una densidad media urbana de 103.2 hab./ha. (CONAPO, 2010b, pp. 38-39). Sin embargo ambas zonas metropolitanas tienen como característica una distribución desigual de su población.

El mapa de densidades habitacionales de Acapulco (Ver Mapa 5) muestra que el crecimiento urbano aleja cada vez más a la población del puerto de la zona de actividades turísticas; diferenciando a los sectores sociales al crear una gran área habitacional al norte y poniente de la ciudad, misma que no disfruta de las condiciones paisajísticas del denominado anfiteatro de la bahía. Áreas populares como Ciudad Renacimiento, El Coloso, Emiliano Zapata y el área comprendida entre el límite de la Zona tradicional y la Laguna de Coyuca presentan las mayores densidades, coincidiendo en el patrón de sectores de bajos ingresos que conforma áreas de alta densidad. En tanto que, las áreas privilegiadas como Las Brisas presentan niveles de densidad bajos. Por el contrario, en la zona tradicional se observa una consolidación de servicios y equipamientos urbanos, ya que tiene alta densidad con un grado de rezago social bajo y alto nivel de equipamiento. En estas áreas la contigüidad de sectores de bajos y altos ingresos podría hacer pensar en una baja segregación, empero, dicha cercanía no implica interacción, ya que existen barreras físicas como muros y fragmentaciones espaciales (Sabatini, 2016, p. 27) como tramas urbanas y agrupamientos de

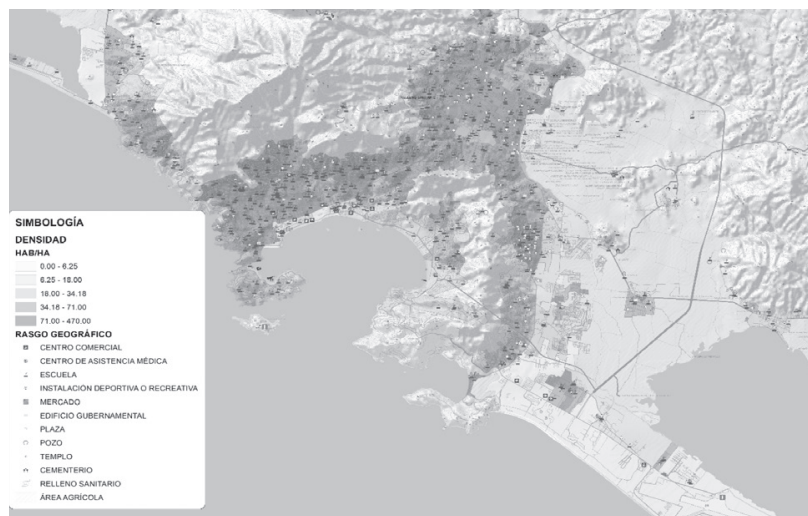

Mapa 5. Zona Metropolitana de Acapulco, densidades habitacionales 2010. Fuente: elaboración propia con base en INEGI (2010)

edificios que impiden la permeabilidad hacia los espacios privilegiados.

Así, para evitar el contacto de los turistas con los habitantes y hacer más veloz su acceso a las zonas turísticas se ha desarrollado infraestructura carretera como el Viaducto Diamante y el Macrotúnel, además de la cercanía del aeropuerto con la zona de desarrollo turístico contemporáneo en la zona diamante. Hacia esta zona del oriente son notorios los macro desarrollos de vivienda, mismos que excluyen otros usos de suelo conformando fraccionamientos habitacionales cerrados, subdivididos en condominios horizontales que cuentan con amenidades como jardines, áreas recreativas y albercas.

Un modelo similar se tiene en la zona periférica de Cancún que implica una gran ocupación de terreno y la conformación de homogéneas ciudades dormitorio. Estos desarrollos no se conurban directamente con la ciudad dejando áreas baldías intermedias, previsiblemente reservadas para la especulación inmobiliaria, lo que genera una expansión difusa del área urbanizada. En el caso de Cancún, estos desarrollos se ubican a lo largo de los ejes conformados por la Avenida Cancún y la Calle 135 al sur de la ciudad. En la zona norte se ubican también grandes desarrollos de vivienda pero destinados a un sector social con ingresos más bajos cercanos al denominado Arco 


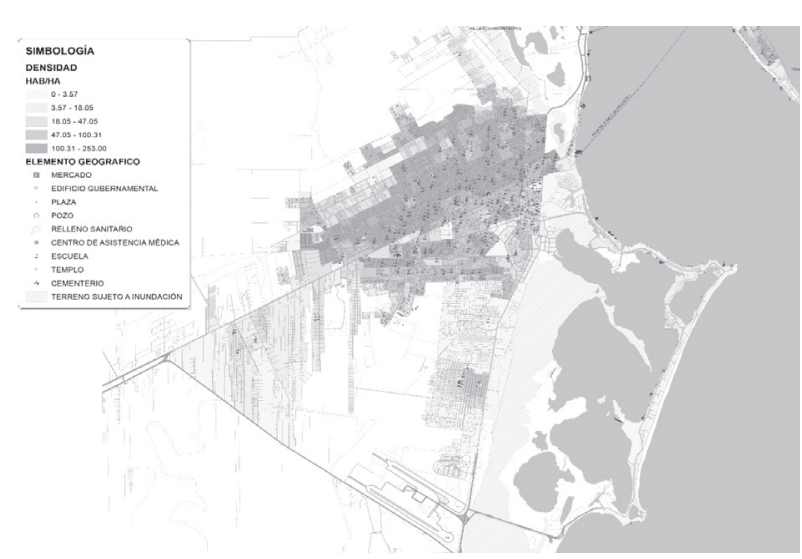

Mapa 6. Zona Metropolitana de Cancún, densidades habitacionales 2010. Fuente: elaboración propia con base en INEGI (2010)

Norte. Asimismo, las zonas populares de Cancún - conocidas como las regiones- se ubican al norte de la ciudad y muestran altas densidades y una traza reticular caracterizada por una dispersión de equipamientos y servicios (Ver Mapa 6).

De manera comparativa, tanto en Acapulco como en Cancún se observa una clara dispersión de equipamientos y servicios. Esto implica que las periferias de ambas ciudades no sólo se conforman de los sectores excluidos en busca de suelo barato, si no que han sido aprovechados por el sector inmobiliario, al ofrecer vivienda destinada a diversos sectores sociales. La característica principal de este modelo es la fragmentación urbana y la expansión en busca de la especulación inmobiliaria. Las zonas urbanas, en muchos casos sin acceso a la playa pero con posibilidad de tener vistas privilegiadas han sido promovidas para desarrollos inmobiliarios verticales para sectores de altos ingresos, como el conformado en Puerto Cancún, a lo largo de la Avenida Bonampak y en Malecón Las Américas. En tanto que en Acapulco se observan estos mismos rasgos en la Zona Dorada y en las cercanías a la zona de Las Brisas. Los vínculos de las elites locales con los equipamientos turísticos son evidentes en su búsqueda de proximidad a las marinas y los campos de golf. Ejemplo de ello, el campo de Golf Tres Vidas en Acapulco y el Pok Ta Pok en Cancún; equipamientos que funcionan también para el turismo de alto nivel de gasto.

\section{Conclusiones}

El turismo ha estado imbricado en la transformación geográfica, económica y social de los territorios, con efectos medioambientales, culturales y socioeconómicos. Por largos periodos, la valorización turística ha estado alimentada por la masificación de la demanda y oferta sobre recursos naturales, culturales e históricos de los espacios. En este proceso la discusión sobre la sustentabilidad del modelo ha estado subordinada a los intereses desarrollistas, comercializadores y de crecimiento del turismo y cuando se le llegó a reconocer careció de un tratamiento integral que incluyera acciones para lograr estándares de vida más equitativos entre los territorios y entre la población que los habita cotidianamente.

Cabe subrayar que, el territorio está en la génesis, experiencia y matices del turismo, es inherente a su naturaleza. De ahí la importancia de entender los procesos, configuraciones y tendencias que constituyen la segregación socio-espacial del turismo que hoy constituyen un dilema para lograr un desarrollo humano sustentable. Por mucho tiempo estuvo presente la idea que la sustentabilidad que acompañaba al desarrollo turístico tenía que ver más con acciones utilitaristas de la calidad del ambiente, que con políticas de calidad de vida de la población local y reducción de la segregación socio-espacial generada en los territorios turísticos. Los intentos de conciliación iniciaron en las décadas finales del siglo XX; momento en el que las discusiones encontradas entre las políticas ambientalistas y las directrices del sistema económico han puesto en conflicto las capacidades ambientales, las políticas de crecimiento económico y las capacidades de equidad y bienestar social y reducción de los niveles de segregación socio-espacial. Con la llegada del siglo XXI se dibujan dimensiones complejas alrededor del ocio relacionadas con el reconocimiento de las polaridades y efectos de segregación sociespaciales en los territorios turísticos.

En particular, el proceso de urbanización de Acapulco y Cancún es diferente por su desarrollo 
histórico, su soporte ambiental y las características particulares de la industria turística en cada destino, el trabajo evidenció que Cancún, como centro turístico competitivo, se constituye a partir de una red de actores, empresas, iniciativas y experiencias que moldean al territorio de acuerdo a la demanda requerida. A partir de estas capacidades ha podido suministrar una variedad de productos y servicios turísticos. Tal reconocimiento le permite crecer, seguir especializándose y posicionándose entre los mejores destinos de playa en México. En tanto que Acapulco mantiene su apuesta sobre el producto de playa y de centros nocturnos, principalmente. Con tales ofertas, Acapulco ha conseguido una demanda más nacional que internacional, mientras en el caso de Cancún es a la inversa.

Además, las redes de Cancún se han multiplicado hacia diferentes niveles del territorio, lo que significa que como centro competitivo mantiene conexiones altamente funcionales. En efecto, los flujos en que interactúa Cancún evidencian la existencia de un territorio eminentemente dinámico. En cambio para Acapulco, la accesibilidad geográfica, la infraestructura turística y los nexos con los que mantiene contacto lo han inclinado hacia el mercado interno, cuyas redes lo terminan moldeando -en promedio- como centro vacacional medianamente competitivo.

Pese a estas diferencias estructurales se pueden nombrar los siguientes patrones de urbanización coincidentes:

a. Existe una marcada segregación activa, funcional, física y virtual de las zonas turísticas con respecto al resto de sus zonas metropolitanas.

b. Ambas ciudades tienen una zona céntrica consolidada en la que interactúan vivienda, equipamientos, comercios y servicios. Estas áreas son ocupadas sobre todo por los habitantes, excluyendo a los turistas por límites físicos como una mala accesibilidad o límites virtuales como una imagen urbana deteriorada, lo que implica que las ciudades turísticas no funcionan como un todo, sino a partir de la articulación de distintas áreas cada una con sus propias dinámicas.

c. Las áreas habitacionales populares, desarrolladas por autoconstrucción, presentan los niveles más altos de densidad habitacional y una mínima dotación de servicios, lo que contrasta con los grandes lotes residenciales de las zonas privilegiadas y el desarrollo de fraccionamientos $\mathrm{y}$ edificios en condominio con equipamientos recreativos y de seguridad, que consumen una gran cantidad de recursos.

d. Para las clases medias se están desarrollando fraccionamientos cerrados con condominios horizontales y para los sectores populares, desarrollos de vivienda de interés social en áreas periféricas, lo que ha generado grandes expansiones de las zonas urbanas sobre áreas de valor ambiental por especulación del suelo periférico.

e. Las nuevas zonas habitacionales son homogéneas y excluyen la presencia de otras actividades y sectores sociales, lo que zonifica especializadamente la ciudad y evita una integración funcional. La vivienda se ve expulsada a las periferias debido al mercado del suelo y el funcionamiento de la industria inmobiliaria.

En un entorno global donde las ciudades presentan procesos de terciarización y desindustrialización, las ciudades turísticas adelantaron muchos de esos procesos, ya que crecieron desde su origen basadas en el sector del comercio y servicios. Sin embargo, con los cambios propiciados por la globalización económica, se observan cambios específicos de esta etapa en la conformación urbana, como la expansión de plazas comerciales y el desarrollo de corredores comerciales y de servicios discontinuos, especializados y con tendencia al agrupamiento en nodos y al desarrollo a lo largo de los ejes de circulación y a la línea costera. En tanto que los sectores de vivienda, comerciales y de servicios vinculados a las zonas turísticas difieren de los utilizados por los habitantes locales, lo que implica un funcionamiento por áreas relativamente independientes, por lo que más que el funcionamiento integral de una ciudad se observa un sistema urbano compuesto de subsistemas homogéneos, 
con funcionalidad interna, pero cada vez menos vinculados al resto de las zonas metropolitanas, lo que está contribuyendo a una mayor diferenciación económica y social.

\section{Referencias bibliográficas}

Aldape, P.G. (2010). La configuración del espacio turístico en Cancún, Quintana Roo, México (Tesis doctoral, Universidad Politécnica de Cataluña, Barcelona, España), Recuperada de: [www.eumed.net/tesis/2010/gap/].

Ayuntamiento de Benito Juárez. (12 de enero de 1993). [Plan Director de Desarrollo Urbano de la Ciudad de Cancún]. Periódico Oficial del Gobierno del Estado de Quintana Roo (Tomo I, No. 2 Extraordinario, 4ta. Época). Chetumal, México.

Ayuntamiento de Benito Juárez. (22 de julio de 2005). Programa de Desarrollo Urbano del Centro de Población de la Ciudad de Cancún. Gaceta Oficial del Gobierno del Estado de Quintana Roo, (Tomo I, No.2, 1a. Época). Chetumal, México.

Calderón, J.R. y Orozco, M.E. (junio-diciembre 2009). Planeación y Modelo Urbano: El Caso de Cancún, Quintana Roo. Quivera, vol. 11, núm. 2, junio-diciembre, pp. 18-34.

Castillo, O. y Villar, A.J. (2011). La conformación del espacio urbano de Cancún: una aproximación al estudio de la segregación socio-espacial. Quivera, vol.13, núm.1, enero-junio, pp. 83-101.

Centro de Investigaciones y Estudios de Turismo de América Latina (CIETAL). (1991). Revista del CIETAL, Año 2, № 1, Centro de Investigaciones y Estudios de Turismo de América Latina, Guerrero, México.

CONEVAL (2010). Consejo Nacional de Evaluación de la Política de Desarrollo Social. Distrito Federal.

CONAPO (2010a). Índice de marginación urbana. México D.F.: Consejo Nacional de Población
CONAPO (2010b). Delimitación de las zonas metropolitanas de México 2010. México: Consejo Nacional de Población Distrito Federal.

Espinosa, H. (enero-junio 2013). El origen del proyecto turístico Cancún, México. Una valoración de sus objetivos iniciales a 42 años de su nacimiento, Liminar. Estudios Sociales y Humanísticos, vol. XI, núm. 1, 2013, pp. 154-167.

INFRATUR (1973). Documento No. 24, Plan Nacional de Turismo 1970. México D.F.: Fondo Nacional de Fomento al Turismo.

FONATUR (1982). Cancún. Un desarrollo turístico en la zona turquesa. Cartografía y Servicios Editoriales. México D.F.: Fondo Nacional de Fomento al Turismo.

Galván, A. (2000). Historia de Acapulco. En: Infoacapulco: innovando al paraíso. Recuperado el 07 de marzo de 2002 de: [http://www.geocities. com/antoniogalvanpuente/historia.html].

Gormsen, E. (1977). El turismo en el Tercer Mundo. En: El turismo como factor de desarrollo regional en México. Informe preliminar sobre un programa de investigación. Main: Geographisches Institut der Johannes Gutenberg University.

INEGI (1960). VIII Censo General de Población, Aguascalientes: Instituto Nacional de Estadística, Geografía e Informática.

INEGI (1987). Cuaderno de información para la planeación. Estado de Guerrero. Aguascalientes: Instituto Nacional de Estadística, Geografía e Informática.

INEGI (2010). Censo de Población y Vivienda 2010. Aguascalientes: Instituto Nacional de Estadística, Geografía e Informática.

INEGI (2016). Marco geoestadístico nacional, Cartografía geoestadística urbana y rural amanzanada. Aguascalientes: Instituto Nacional de Estadística, Geografía e Informática.

Martí, F. (1985). Cancún: Fantasía de Banqueros. México: Editorial Uno. 
McCoy, C.E. (2015). Propuesta para recuperar la perspectiva de destino turístico sustentable con base en el análisis de su desempeño y evolución: Caso Cancún (Tesis doctoral en Desarrollo Económico Regional y Sectorial Estratégico, Universidad Popular Autónoma del Estado de Puebla, México).

Ochoa, O.M. (1964). Guerrero: análisis de un estado problema. Ciudad de México: Trillas.

Pérez, E. (2010). Segregación socio-espacial en ciudades turísticas, el caso de Puerto Vallarta, México. Región y Sociedad, Vol. XXII, No. 49, pp. 143-176.

Sabatini, F. (2015). La ruptura del patrón de segregación y su significado teórico y práctico. En: Aguilar, A. y Escamilla, I (Coords.) Segregación urbana y espacios de exclusión. Ejemplos de México y América Latina. México: Porrúa.

Sabatini, F. (2016). “¿Podemos construir ciudades menos segregadas?. En Álvarez, L. y Delgado,
G., Leal, A. (Coords.). Los desafíos de la ciudad del siglo XXI. México: Universidad Nacional Autónoma de México.

Schornick, F. (1977). El turismo como factor de desarrollo regional en México. Informe preliminar sobre un programa de investigación. Geographisches Institut der Johannes Gutenberg University. Mainz, Alemania.

Schteingart, M. (2015). La división social del espacio en ciudades mexicanas: Un balance explicativo desde una perspectiva latinoamericana. En: Aguilar, A. y Escamilla, I (Coords.) Segregación urbana y espacios de exclusión. Ejemplos de México y América Latina. México: Porrúa.

Segrado, R., González, A., Arroyo L. y Palafox, A. (2010). El desarrollo de Cancún, analizado desde la teoría de los sistemas complejos. Gestión Turística (núm. 14, julio-diciembre). pp. 9-32. 